\title{
Activity of Clinically Relevant Antimalarial Drugs on Plasmodium falciparum Mature Gametocytes in an ATP Bioluminescence "Transmission Blocking" Assay
}

\author{
Joël Lelièvre ${ }^{1 * 9}$, Maria Jesus Almela ${ }^{19}$, Sonia Lozano ${ }^{1}$, Celia Miguel ${ }^{1}$, Virginia Franco ${ }^{1 \times}$, Didier Leroy ${ }^{2}$, \\ Esperanza Herreros ${ }^{1 *}$
}

1 GlaxoSmithKline R\&D, Tres Cantos Medicine Development Campus, Malaria Discovery Performance Unit, Madrid, Spain, 2 Medicines for Malaria Venture, Geneva, Switzerland

\begin{abstract}
Background: Current anti-malarial drugs have been selected on the basis of their activity against the symptom-causing asexual blood stage of the parasite. Which of these drugs also target gametocytes, in the sexual stage responsible for disease transmission, remains unknown. Blocking transmission is one of the main strategies in the eradication agenda and requires the identification of new molecules that are active against gametocytes. However, to date, the main limitation for measuring the effect of molecules against mature gametocytes on a large scale is the lack of a standardized and reliable method. Here we provide an efficient method to produce and purify mature gametocytes in vitro. Based on this new procedure, we developed a robust, affordable, and sensitive ATP bioluminescence-based assay. We then assessed the activity of 17 gold-standard anti-malarial drugs on Plasmodium late stage gametocytes.

Methods and Findings: Difficulties in producing large amounts of gametocytes have limited progress in the development of malaria transmission blocking assays. We improved the method established by Ifediba and Vanderberg to obtain viable, mature gametocytes en masse, whatever the strain used. We designed an assay to determine the activity of antimalarial drugs based on the intracellular ATP content of purified stage IV-V gametocytes after $48 \mathrm{~h}$ of drug exposure in 96/384-well microplates. Measurements of drug activity on asexual stages and cytotoxicity on HepG2 cells were also obtained to estimate the specificity of the active drugs.

Conclusions: The work described here represents another significant step towards determination of the activity of new molecules on mature gametocytes of any strain with an automated assay suitable for medium/high-throughput screening. Considering that the biology of the forms involved in the sexual and asexual stages is very different, a screen of our 2 million-compound library may allow us to discover novel anti-malarial drugs to target gametocyte-specific metabolic pathways.
\end{abstract}

Citation: Lelièvre J, Almela MJ, Lozano S, Miguel C, Franco V, et al. (2012) Activity of Clinically Relevant Antimalarial Drugs on Plasmodium falciparum Mature Gametocytes in an ATP Bioluminescence "Transmission Blocking" Assay. PLoS ONE 7(4): e35019. doi:10.1371/journal.pone.0035019

Editor: Ross Frederick Waller, University of Melbourne, Australia

Received October 27, 2011; Accepted March 8, 2012; Published April 13, 2012

Copyright: (c) 2012 Lelièvre et al. This is an open-access article distributed under the terms of the Creative Commons Attribution License, which permits unrestricted use, distribution, and reproduction in any medium, provided the original author and source are credited.

Funding: The authors have no support or funding to report.

Competing Interests: The authors have read the journal's policy and have the following conflicts: JL SL MJA EH CM are employees of GlaxoSmithKline (GSK) and work was funded by GSK under a general research program. DL is employee of Medicines for Malaria Venture (MMV). This does not alter the authors' adherence to all the PLoS ONE policies on sharing data and materials. Neither GSK nor MMV have influenced or biased the content of this work.

*E-mail: Joel.I.lelievre@gsk.com (JL); esperanza.a.herreros@gsk.com (EH)

9 These authors contributed equally to this work.

a Current address: Centro de Biología Molecular (UAM-CSIC), Campus de la Universidad Autónoma de Madrid, Madrid, Spain

\section{Introduction}

Recent years have witnessed renewed impetus for malaria control but this disease is still leading to nearly 1 million deaths annually. This is why the Malaria Eradication Research Agenda (malERA, http://malera.tropika.net) initiative, created in 2007, re-established the long-term goal of malaria eradication. Spread and maintenance of the malaria parasites rely on their transmission between humans and Anopheles. Attempts at eradication should therefore include transmission-blocking approaches aiming at identification of therapies capable of eliminating Plasmodium gametocytes, the sexual forms of the parasite. To test potential anti-gametocyte drugs, both an improved gametocyte production method and a reliable assay to assess activity of compounds against mature gametocytes are required.

The biology of gametocytes is complex and consists of five morphologically identifiable stages: stages I to IV (immature gametocytes) sequestered into human tissues; and stage $\mathrm{V}$, the infectious sexual form that circulates in the blood-stream. Despite some attempts to gain insights into the biology of the parasite's gametocytes and to identify potential drug targets within their proteomes [1], identification of new molecules blocking the production of these forms relied mainly on phenotypic assays and recently on a small number of novel assays using ATP 
detection [2], flow cytometry [3], stage-specific markers such as Pfs 16 [4,5] or both of the latter two methods [6].

Moreover, the renewed interest in parasite transmission has highlighted a crucial need for additional tools. Difficulties in producing large amounts of this form have limited research progress in the development of blocking transmission assays. Using the method of Trager and Jensen, Smalley was the first to attempt to obtain sexual forms of the parasite, but these gametocytes were not, or were rarely and unpredictably functional, even when they were allowed to develop for a longer time [7]. In 1981, Ifediba and Vanderberg improved the method by adding hypoxanthine to the culture medium and obtained mature $P$. falciparum gametocytes [8]. It later became possible to remove the asexual forms in most gametocyte production protocols by introducing N-acetyl-Dglucosamine (GLcNA), a sugar that completely blocks invasion of the erythrocyte by this parasite but does not affect gametocyte maturation [9]. More recently, Mann found that addition of bistratene $\mathrm{A}$, a protein kinase $\mathrm{C}$ inhibitor, also inhibited merozoite invasion and reportedly induced gametocytogenesis [10]. Moreover, Fivelman et al [11] established a useful but complex protocol to obtain gametocytes reaching different maturation stages. All of these methods have a low production rate and suffer from costs and reproducibility problems because culture medium is currently supplemented with human serum in different proportions.

Currently, we lack effective activity assays against late stage gametocytes. The gold standard test relies on dose-response measurement by manual microscopic counting of the number of gametocytes after drug exposure at different concentrations. This method is time-consuming and subject to human error. Flow cytometry has been proposed to assess the in vitro gametocytocidal activities of potential anti-plasmodial drugs [3] but each well has to be processed on costly equipment. Whilst an improvement on the light microscopy method, the use of cytometry does not allow for large-scale assessment of compound activity on late-stage gametocytes. Another promising assay, which has recently been developed [2], is based on detection of fluorescence of reduced alamar blue. Nevertheless, several important drawbacks to its routine use have been reported [12] such as the amount of gametocytes required, and the targeted stage which is not confined to mature gametocytes. The ATP bioluminescent assay remains a gold standard to explore parasite viability. ATP plays a central role in energy exchanges in biological systems (both eukaryotic and prokaryotic), serves as the main donor of free energy, and is produced in all metabolically active cells. This parameter is currently used as a tool to assess the functional integrity of living cells. Injury and death result in a rapid decrease in cytoplasmic ATP, [13] which therefore provides a reliable platform to test the effect of small molecules on the ability of gametocytes to develop. An assay based on ATP has in fact been published recently. However, methodological flaws can be noted; a crucial one is the use of frozen gametocytes [14].

We report an innovative solution to produce and purify mature (stage IV-V) gametocytes and to develop and validate a method based on bioluminescence-mediated detection of ATP as the preferred measure of gametocyte viability. This assay has been validated successfully and for the first time provides extensive activity data of 17 clinically relevant antimalarial drugs.

\section{Results}

\section{Gametocyte production protocol}

Capitalizing on previous studies, we developed a method which overcomes some significant constraints. We started to use lipidrich bovine serum albumin concentrate (AlbuMAX II) instead of the standard supplement, human serum. AlbuMAX is much cheaper and avoids variability between the different serum batches and donors, thus increasing the reliability of the assay. We also kept adding hypoxanthine to the basal medium throughout the process and added $\mathcal{N}$-acetyl-D-glucosamine and bistratene A during the last phase of gametocyte differentiation to obtain approximately $1-2 \%$ gametocytemia (stage IV and V) between day 15 and day 18 .

Under these conditions, different strains of $P$. falciparum (3D7A, W2, NF54, Dd2 and 3D7HT-GFP) were tested to determine which one was the most appropriate. Differences in gametocyte production were seen among these strains. The $P$. falciparum 3D7HT-GFP strain [15], that constitutively expresses green fluorescent protein (GFP), showed the highest sexual differentiation ratio and the greatest ability to mature and was therefore used to set up the assay. The gametocytogenesis profile of the 3D7HTGFP strain is shown in Figure 1.

\section{Purification process}

Our gametocyte culture is composed of $1-2 \%$ mature gametocytes but also 98-99\% red blood cells (RBCs), which contain a significant intracellular ATP concentration that interferes with the readout. It was therefore necessary for RBCs to be removed from the culture. Stage IV-V gametocyte cultures were enriched on a Nycoprep cushion, based on density differences between gametocytes and RBCs. Most RBCs were depleted, but an accurate quality control of samples showed that an unacceptable number remained. An additional purification step, consisting of magnetic column separation, was thus performed. The ability of all Plasmodium erythrocytic species to degrade hemoglobin (a Fe(II) diamagnetic complex) to hemozoin (a $\mathrm{Fe}$ (III) paramagnetic complex) [16] was used, making discrimination between retained gametocytes and eluted RBC possible when the magnetic field was applied. We thus managed to obtain a culture containing more than $70 \%$ stage IV-V gametocytes (Fig. 2). We were then concerned by the viability of gametocytes after this two-step purification. Intracellular ATP

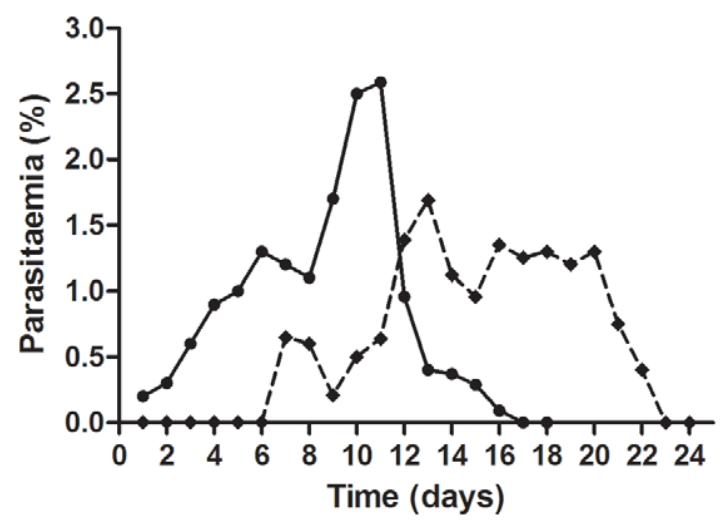

Figure 1. Kinetics of parasitaemia (asexual stages only, black dots) and gametocytaemia (stages 1-5; black squares) by microscopic observation, represented as a percentage of total erythrocytes. The number of asexual forms increased up to maximum parasitaemia on day 11. From that time onwards, a rapid decrease occurred leading to a parasitaemia (asexual stages) close to zero on day 17. Sexual forms were first detected on day 7 , and gametocytaemia reached a peak of $1.69 \%$ on day 13 . The gametocytes, initially in early forms, developed into mature stages reaching stages IV and $\mathrm{V}$ from day 15 to 20 . After day 15 , more than $70 \%$ of the gametocytes are in stage IV-V.

doi:10.1371/journal.pone.0035019.g001 


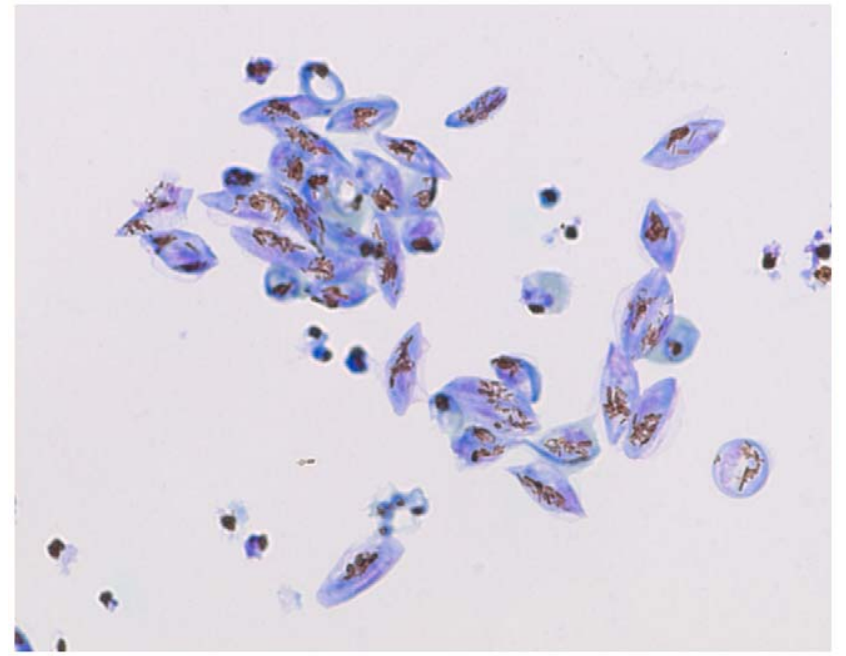

Figure 2. Giemsa-stained culture of gametocytes on day 15 after both Nycoprep and magnetic purification steps, $(1000 \times)$. doi:10.1371/journal.pone.0035019.g002

level, morphology, internal motility and response to exflagellation stimuli were therefore all checked (data in annex) at $\mathrm{T}=0 \mathrm{~h}$ and $\mathrm{T}=48 \mathrm{~h}$, and the results showed that our purification approach did not significantly affect either viability or exflagellation of gametocytes throughout the purification procedure.

\section{Assay development and validation}

Following successful mass production of viable gametocytes, an assay for large-scale testing of the activity of compounds against stage IV-V gametocytes, by measuring ATP levels after drug exposure, was developed. Gametocytes ATP intracellular levels were measured by the luciferin-luciferase methodology.

Firstly, to assess the robustness and the quality of the ATP bioluminescence assay we calculated the $Z^{\prime}$-factor, the screening window coefficient (calculated with the control data) [17]. Values between 0.5 and 1.0 indicate excellent assay quality. The $Z^{\prime}$-factor for the assay ( \pm standard deviation) was $0.68( \pm 0.02)$. To determine whether ATP quantification provided a readout that was directly proportional to the number of viable parasites, we compared microscopy counts with ATP levels. This is important to confirm the reliability of the assay. A high degree of correlation was observed $\left(R^{2}=0.99\right)$ as shown in Figure 3.

Then, to validate the assay, 6 common anti-plasmodial drugs such as epoxomicin, dihydroartemisinin, artesunate, primaquine, chloroquine and methylene blue were tested. Dose-response curves and $\mathrm{IC}_{50}$ values determined by quantification of the intra cellular ATP were compared to those obtained in parallel using the standard method of microscopic enumeration of gametocytes on stained smears after drug exposure. Cytotoxicity studies were also carried out to investigate the corresponding selectivity ratio (activity in HepG2 cells versus activity against $P$. falciparum gametocytes)

The first compound evaluated, the proteasome inhibitor epoxomicin, is known to have a very potent effect on gametocytes [2]. In the present study, it also exhibited a very high activity in both the ATP bioluminescence assay $(0.42 \mathrm{nM})$ and microscopically $(5.2 \mathrm{nM})$. Nevertheless, $\mathrm{IC}_{50}$ values $(3 \mathrm{nM})$ in HepG2 cells were in the same range, suggesting a total lack of selectivity. Inhibition of proteasome activity has a toxic effect on the parasite, but this function is also essential in mammalian cells and, considering the difference of cytotoxicity exhibited by epoxomicin

\section{P. falciparum 3D7HT-GFP}

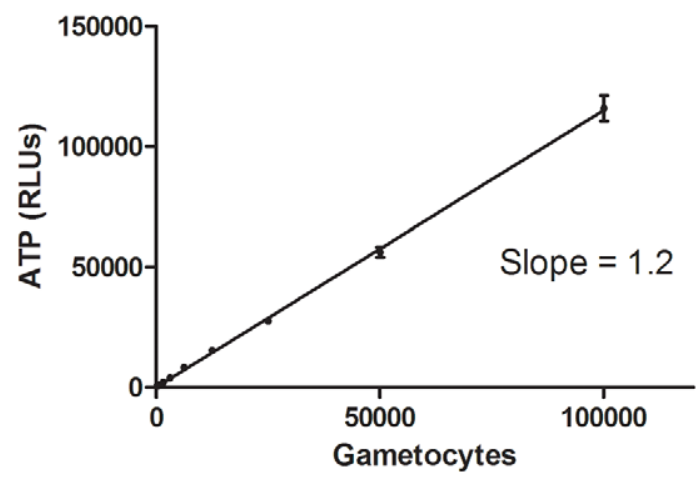

Figure 3. ATP level per gametocyte number $\left(R^{2}=0.99\right)$ counted with the luminometer after serial dilution. Gametocytes were counted using a Neubauer chamber. Each point represents mean values of 3 replicates \pm SD.

doi:10.1371/journal.pone.0035019.g003

on the HepG2 cell line and on mouse 3T3 or human A549 cells [18], its use may have to be reconsidered.

The two artemisinin derivatives used in our study, dihydroartemisinin (DHA) and artesunate, had no particular activity against mature gametocytes with $\mathrm{IC}_{50}$ values of $3.56 \mu \mathrm{M}$ and $10.83 \mu \mathrm{M}$ respectively. Activity of artemisinins against these forms is still controversial as very few data have been reported. The effect of artesunate was described in only two studies that found $\mathrm{IC}_{50}$ values of $108 \mathrm{nM}$ [19] and $0.1 \mathrm{ng} / \mathrm{mL}$ [20]. A single article, describing a new flow cytometry assay [3], demonstrated an $\mathrm{IC}_{50}$ of $1 \mu \mathrm{M}$ for artemisinin on late gametocytes and its metabolite, DHA, was found to inhibit gametocytes by $70.4 \%$ at $1 \mu \mathrm{M}$ [2]. It is well known that treatment of Plasmodium with artemisinin derivatives is associated with lower rates of gametocyte carriage [21,22] but there is a huge gap in in vitro knowledge and additional work is required. Interestingly, the two artemisinin derivatives in the present study showed a high selectivity for the target in asexual stages as $\mathrm{IC}_{50}$ values obtained in HepG2 cells exceeded $50 \mu \mathrm{M}$.

Primaquine has been reported to selectively destroy the inner structure of P.falciparum mitochondria. The mechanism by which primaquine causes its effects on mitochondria remains unclear. It is assumed that primaquine activity depends to a large extent on the formation of metabolites which are more active than the parent compound [23]. This 8-aminoquinoline has long been known to reduce the prevalence of circulating gametocytes in the peripheral bloodstream of parasitemic individuals and to prevent exflagellation among gametocytes present in the test [24,25]. The $\mathrm{IC}_{50}$ on late gametocytes $(20.9 \mu \mathrm{M})$ and asexual forms $(>10 \mu \mathrm{M})$ described in this study are in accordance with current literature. Due to the absence of the active metabolites involved in its mechanism of action, primaquine remains inactive in vitro. The related 4-aminoquinolines, including chloroquine, affect $P$. falciparum immature gametocytes but not mature gametocytes [26]. Chloroquine kills the Plasmodium parasite by inhibiting hemozoin formation and glutathione degradation. Absence of these metabolic reactions at stages IV and V would account for the inactivity of chloroquine reported by Smalley et al. [26] The $\mathrm{IC}_{50}$ of chloroquine observed with our ATP bioluminescence assay $(23.47 \mu \mathrm{M})$ agreed with the values reported in the literature.

Methylene blue $(\mathrm{MB})$ has recently been reconsidered as a useful antimalarial drug [4] and has been explored in combination with $\mathrm{CQ}$ and artemisinin derivatives [27]. $\mathrm{MB}$ was identified as a specific inhibitor of $P$. falciparum glutathione reductase, and blocks 
heme polymerization within the food vacuole, is active against all asexual blood stages. Promising data have been reported in clinical trials [28] and, to date, only one publication has shown the in vitro activity of $\mathrm{MB}$ in mature gametocytes [4]. Our study evaluated for the first time the $\mathrm{IC}_{50}$ of $\mathrm{MB}$ on stage $\mathrm{IV}-\mathrm{V}$ gametocytes $(0.49 \mu \mathrm{M})$ and the $\mathrm{IC}_{50}$ on the HepG2 cell line $(6.52 \mu \mathrm{M})$, and found good activity but quite high cytotoxicity.

As mentioned earlier, it was essential to determine the activity on mature gametocytes using the gold standard microscopy-based parasite enumeration to validate the assay. As an example, the dose-response curves obtained for both artesunate and methylene blue were represented in Figure 4 and all the $\mathrm{IC}_{50}$ results are summarized in Table 1 . Microscopy examination showed $\mathrm{IC}_{50}$ values comparable to those obtained with the ATP bioluminescence assay in all cases. Differences were expected because one of the drawbacks of the microscopic method is that all parasites are counted, including dead parasites that represent false positives. Even with this shift, the active/inactive profile observed in the dose-response curves calculated from microscopic observations supported the results found with the ATP bioluminescence assay and thus validated this technique.

\section{From validation to test}

After validation had been performed and based on the differences in mechanisms of action, 10 other drugs currently in use or in clinical development were tested against mature gametocytes using the ATP bioluminescence assay.

The activity against asexual stages was also determined (Table 2) with the reference strain line 3D7A and the 3D7HT-GFP strain to confirm that they both have the same behavior. The activity against mature gametocytes was done using the 3D7HT-GFP strain. Finally, cytotoxicity in the human cell line HepG2 was assessed.

Among the 10 newly tested compounds, quinine and mefloquine, were first investigated. As with other quinoline anti-malarial drugs, the mechanism of action of quinine has not been fully elucidated. The most widely accepted hypothesis about quinine action is based on data for the closely related drug, mefloquine $[29,30]$. These data suggest that its main mode of action may be to inhibit hemoglobin ingestion by blocking the endocytotic process [31]. Although they are active against asexual blood stages of chloroquine-resistant (CQR) and sensitive (CQS) P. falciparum strains $[32,33,34]$, neither quinine $\left(\mathrm{IC}_{50}\right.$ above $\left.50 \mu \mathrm{M}\right)$ nor mefloquine $\left(\mathrm{IC}_{50}=4.7 \mu \mathrm{M}\right)$ showed good activity on late game- tocytes. Moreover, the selectivity ratio of mefloquine (2.34) was quite narrow $\left(\mathrm{IC}_{50}\right.$ of $11 \mu \mathrm{M}$ in HepG2 cells versus $\mathrm{IC}_{50}$ of $4.9 \mu \mathrm{M}$ in gametocytes)

Several of the compounds that act on hemoglobin metabolism were also evaluated; halofantrine, lumefantrine and isoquine. The exact mechanism by which halofantrine and lumefantrine exert their anti-malarial effect is not well defined. However, available data suggests that halofantrine binds to plasmepsin, a hemoglobindegrading enzyme and to haematin in vitro and lumefantrine inhibits the formation of $\beta$-haematin by forming a complex with haemin. Isoquine, on the other hand, was developed as a potentially safer alternative to amodiaquine, an effective but toxic substitute of chloroquine. Halofantrine, isoquine and lumefantrine (used in the "co-artemether" combination) are active on asexual blood stages of CQR strains $[32,35,36]$ but no significant activity on mature gametocytes was detected $\left(\mathrm{IC}_{50}=6.7 ; 28.5\right.$ and $>50 \mu \mathrm{M}$, respectively) [37]. Lumefantrine and halofantrine were less cytotoxic than isoquine in vitro with an $\mathrm{IC}_{50}$ higher than $50 \mu \mathrm{M}$ for both, compared to $17.07 \mu \mathrm{M}$ for isoquine.

Pyronaridine-artesunate is a promising new artemisinin-based combination therapy for the treatment of uncomplicated falciparum malaria. Pyronaridine is also thought to inhibit parasite development because of its ability to inhibit $\beta$-haematin formation. It has been shown to have a high in vitro activity against chloroquinesensitive and chloroquine-resistant strains of P. falciparum [38]. Moreover, some activity against gametocytes has been reported [39] but what was probably seen was an effect on young gametocytes because the $\mathrm{IC}_{50}$ for mature gametocytes was approximately $3.25 \mu \mathrm{M}$ with the ATP bioluminescence assay.

The antifolate family is an important group of antimalarials of which pyrimethamine is a crucial member. The compound was introduced here as an internal control as the strain $P$. falciparum 3D7HT-GFP contains a selection cassette giving resistance to drugs acting on DHFR. The drug, as expected, did not have any effect on mature gametocytes of this resistant line.

It should be noted that atovaquone, which is often used today in the atovaquone-proguanil combination (Malarone), showed very strong in vitro activity against asexual erythrocytic $P$. falciparum cultures $\left(\mathrm{IC}_{50}=1.8 \mathrm{nM}\right)$ but had no activity against gametocytes according to our ATP assay $\left(\mathrm{IC}_{50}=16.10 \mu \mathrm{M}\right)$ and previous reports [19]. It has been shown that atovaquone does inhibit transmission $[37,40]$ but has no effect on mature gametocytes [41]. No cytotoxic effects were detected at the maximum solubility of atovaquone in culture media.

\section{artesunate}

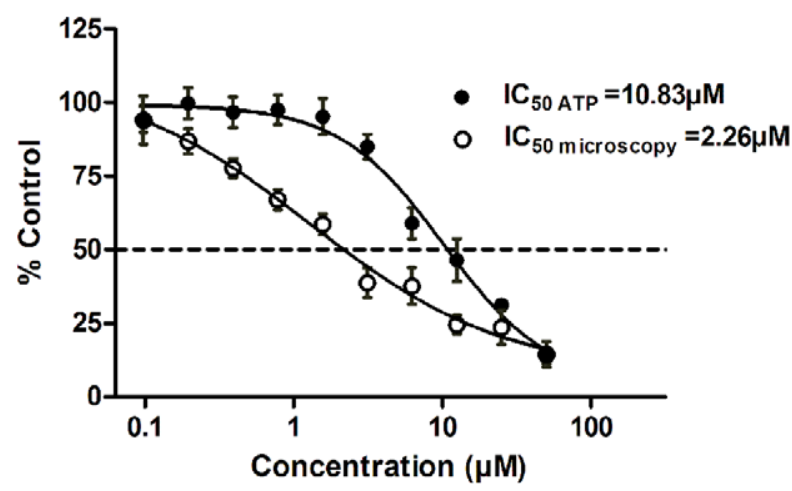

methylene blue

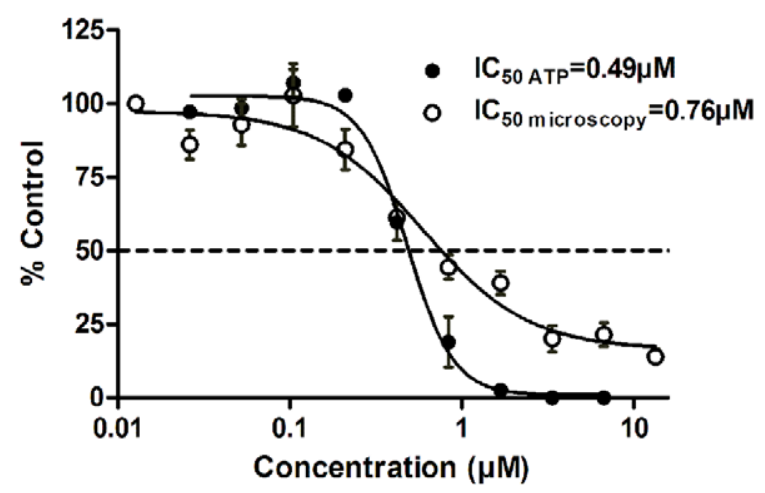

Figure 4. Comparison of the dose-response curves of artesunate and methylene blue obtained with the ATP bioluminescence assay (black dots) or by microscopic counting (open circles). Each point represents mean values of 4 replicates \pm SD. doi:10.1371/journal.pone.0035019.g004 
Table 1. Comparison of the activity against mature gametocytes $\left(\mathrm{IC}_{50}\right)$ using either the ATP bioluminescence assay or the microscopical enumeration.

\begin{tabular}{lll}
\hline & & \\
\hline Compounds & $\begin{array}{l}\text { IC } \\
\text { Microscopy examination }\end{array}$ & $\begin{array}{l}\text { IC }_{50} \text { gametocytes }(\mu \mathrm{M}) \\
\text { ATP method }\end{array}$ \\
\hline Chloroquine & 28.4 & 23.47 \\
Artesunate & 2.26 & 10.83 \\
Methylene blue & 0.76 & 0.49 \\
Primaquine & 87.9 & 20.9 \\
Dihydroartemisinin & 2.47 & 3.56 \\
Epoxomicin & 0.0052 & 0.00042 \\
\hline doi:10.1371/journal.pone.0035019.t001 &
\end{tabular}

Use of antibiotics was also investigated. Cycloheximide is an inhibitor of protein biosynthesis in eukaryotic organisms that interferes with the translocation step in protein synthesis. Experimental evidence of antimalarial activity is available for this drug [42] but it showed low activity when tested on stage IV-V gametocytes $\left(\mathrm{IC}_{50}=6.23 \mu \mathrm{M}\right)$, as described previously [43]. Asexual forms of the parasite may also be killed by targeting the apicoplast. Azithromycin is a macrolide antibiotic derived from erythromycin. It is not yet licensed for use as an antimalarial agent but has shown promising activity against $P$. falciparum in vitro [44]. This drug appeared to have some effect upon parasite development in the mosquito stage [45] but had no effect upon mature gametocytes, in the present study $\left(\mathrm{IC}_{50}=28.45 \mu \mathrm{M}\right)$ and in other experiments [46].

\section{Discussion}

The eradication of malaria will remain an elusive goal unless drugs are found that either massively reduce or, even better, fully inhibit disease transmission. The lack of tools to assess the activity of compounds against the key actors, mature gametocytes, is the real problem at present. Some attempts have been made $[2,3,4,14]$, but published methods are still labor-intensive, costly and/or complex, making development of such a large-scale screening difficult.

Table 2. In vitro activity of the compounds against asexual stages, mature gametocytes and assessment of cytotoxicity in the HepG2 cell line.

\begin{tabular}{|c|c|c|c|c|}
\hline \multirow[t]{2}{*}{ Compounds } & \multicolumn{4}{|l|}{$I C_{50}(\mu M)$} \\
\hline & $\begin{array}{l}\text { Activity against asexual } \\
\text { forms (3D7A) }\end{array}$ & $\begin{array}{l}\text { Activity against asexual forms } \\
\text { (3D7HT-GFP) }\end{array}$ & $\begin{array}{l}\text { Activity against mature } \\
\text { gametocytes IV-V }\end{array}$ & Cytotoxicity \\
\hline \multicolumn{5}{|l|}{ Quinolines } \\
\hline Chloroquine & $0.006 \pm 0.0002$ & $0.007 \pm 0.002$ & $23.47 \pm 2.66$ & $51.84 \pm 7.86$ \\
\hline Primaquine & $>10$ & $>10$ & $20.90 \pm 4.65$ & $>50$ \\
\hline Isoquine & $0.009 \pm 0.0016$ & $0.0044 \pm 0.0032$ & $28.5 \pm 2.12$ & $17.07 \pm 6.3$ \\
\hline Pyronaridine & $0.0045 \pm 0.0015$ & $0.00080 \pm 0.0001$ & $3.25 \pm 0.92$ & $5.95 \pm 0.92$ \\
\hline \multicolumn{5}{|c|}{ Artemisinin Endoperoxides } \\
\hline Artesunate & $0.037 \pm 0.025$ & $0.054 \pm 0.03$ & $10.83 \pm 3.7$ & $>50$ \\
\hline Dihydroartemisinin & $0.0028 \pm 0.0007$ & $0.013 \pm 0.003$ & $3.56 \pm 0.95$ & $>50$ \\
\hline \multicolumn{5}{|l|}{ Amino alcohols } \\
\hline Quinine & $0.066 \pm 0.018$ & $0.106 \pm 0.040$ & $>50$ & $>50$ \\
\hline Mefloquine & $0.026 \pm 0.017$ & $0.028 \pm 0.0014$ & $4.70 \pm 2.10$ & $11 \pm 0.26$ \\
\hline Lumefantrine & $0.0032 \pm 0.00014$ & $0.00165 \pm 0.00007$ & $>50$ & $>50$ \\
\hline Halofantrine & $0.0011 \pm 0.0005$ & $0.0012 \pm 0.0005$ & $6.70 \pm 0.42$ & $>50$ \\
\hline \multicolumn{5}{|l|}{ DHFR-TS inhibitors } \\
\hline Pyrimethamine & $0.0025 \pm 0.0009$ & $33.66 \pm 6.72^{*}$ & $>50$ & $>50$ \\
\hline \multicolumn{5}{|c|}{ Hydroxy-naphthoquinones } \\
\hline Atovaquone & $0.0007 \pm 0.0004$ & $0.0018 \pm 0.0002$ & $16.10 \pm 2.12$ & $>40^{\mathrm{a}}$ \\
\hline \multicolumn{5}{|l|}{ Proteasome inhibitors } \\
\hline Epoxomicin & $0.008 \pm 0.001$ & $0.0098 \pm 0.0017$ & $0.00042 \pm 0.00011$ & $0.003 \pm 0.0005$ \\
\hline \multicolumn{5}{|l|}{ Dyes } \\
\hline Methylene blue & $0.011 \pm 0.008$ & $0.010 \pm 0.0018$ & $0.49 \pm 0.16$ & $6.52 \pm 1.31$ \\
\hline \multicolumn{5}{|c|}{ Protein synthesis inhbitors } \\
\hline Azythromycin & $>10$ & $>10$ & $28.45 \pm 0.64$ & $>50$ \\
\hline Cycloheximide & $0.198 \pm 0.0017$ & $0.194 \pm 0.0077$ & $6.23 \pm 2.46$ & $2.95 \pm 0.35$ \\
\hline
\end{tabular}

Inhibition of uptake of a radiolabeled nucleic acid precursor by the parasites served as the indicator of antimalarial activity against asexual stages while the activity on mature gametocytes was determined with the ATP bioluminescence assay. Finally, cytotoxicity was assessed using the short-term resazurin-based reductase assay. All the results are expressed as the average value of the $\mathrm{IC}_{50} \pm$ standard deviation (3-6 independent experiments).

alimit of solubility.

*resistance to pyrimethamine due to a selection cassette in the episome (containing the GFP gene).

doi:10.1371/journal.pone.0035019.t002 
Compared to previous methods that assessed the activity of new chemical entities against mature gametocytes, the technique that we have devised has several advantages. This ATP bioluminescence assay is highly sensitive; the luminometric technique is able to detect a low number of cells $\left(1 \times 10^{4}\right.$ per well in a 384 -well plate $)$ even if these cells display a low metabolic activity. This method measures the whole well in the microtiter plate, representative of a population of parasites. When samples are analyzed by microscopy, only an aliquot is taken from the well to prepare a smear and only some fields are counted at random. In addition, our methodology does not rely on operator expertise and is therefore not subject to person-to-person variability. Uncertainty about the viability of parasites when counting is overcome by this new method as only metabolically active parasites are detected. Reading of luminescence is fast, requiring a minimum number of handling steps and providing results within 15 minutes. The plate is read instantaneously and there is no need for timeconsuming incubation, as required by other techniques (e.g. alamar blue). An important advantage is that the method can be used to directly evaluate the activity on any parasite strain or clinical isolates as it does not require the use of any parasite line transformed with reporter genes. Moreover, this system is amenable to adaptation to 384-well plates allowing the testing of a higher number of compounds than with traditional manual counting or the flow cytometry technique.

In summary, the work described here provides, for the first time, data on the activity of all clinically relevant antimalarial drugs against mature gametocytes and represents a significant step towards development of a high-content method suitable for highthroughput screening of new molecules at these stages. Considering that the biology of the forms involved in the sexual and asexual stages is very different, a large-scale high-throughput screen using the GlaxoSmithKline 2 million-compound library may allow us to discover innovative anti-malarial drugs to target novel transmission-specific metabolic pathways.

\section{Methods}

\section{Parasites and Cultures}

Three strains of Plasmodium falciparum, W2-Indochina (MR4, MRA-157), 3D7A (MR4, MRA-151) and the 3D7HT-GFP strain [15], kindly provided by Robert E. Sinden, were cultured using a method modified from that of Trager and Jensen [47] in a $5 \%$ $\mathrm{CO}_{2} / 5 \% \mathrm{O}_{2}$ atmosphere at $37^{\circ} \mathrm{C}$ [48].

\section{Gametocytogenesis}

Gametocyte cultures of each strain were initiated as described by Ifediba and Vanderberg [8], but with the following minor variations: On day 0 , cultures were synchronized at the ring stage by lysis of the flask pellet with 5 volumes of $5 \%$ sorbitol for $10 \mathrm{~min}$ at $37^{\circ} \mathrm{C}$. Cultures were then initiated at $0.2 \%$ parasitaemia and $12 \%$ hematocrit in a $10 \mathrm{~mL}$ volume. The culture was first incubated in RPMI 1640 (Gibco) supplemented with hypoxanthine (Sigma-Aldrich), sodium bicarbonate (Sigma-Aldrich) and $15 \%$ AlbuMAX II solution. On day 8, the volume was doubled and the concentration of AlbuMAX was reduced to $10 \%$. Finally, on day 12 , cultures were treated with $50 \mathrm{mM} \mathcal{N}$-acetyl-Dglucosamine (GLcNA, Sigma-Aldrich) and $50 \mathrm{ng} / \mathrm{mL}$ of bistratene A for 3-5 days to remove asexual forms. Medium was changed daily throughout the process. To assess gametocytogenesis curves (Fig. 1), the percentage of asexual forms and gametocytes were counted on Giemsa-stained smears.

\section{Purification}

On day 15 (Fig. 1), the majority of the gametocytes were stage IV-V. At this point, parasites were concentrated with NycoPrep $^{\text {TM }} 1.077$ cushions (Axis-Shield). The culture pellet was washed, resuspended in $10 \mathrm{~mL}$ of medium and placed slowly onto $5 \mathrm{~mL}$ of Nycoprep cushion in a $15 \mathrm{~mL}$ Falcon tube. The tube was centrifuged at $800 \mathrm{~g}$ for 20 minutes. The band was collected, washed, and spun down and the pellet was resuspended in $5 \mathrm{~mL}$ of medium. During the entire procedure the gametocytes were maintained at $37^{\circ} \mathrm{C}$. A Giemsa-stained smear was prepared at this point. The solution containing the gametocytes was then loaded onto a LS-Column (Miltenyi Biotech) to carry out the second purification step using a VarioMACS magnetic separator (Miltenyi Biotech). The column was washed with $5 \mathrm{~mL}$ of medium to elute the remaining RBCs and, finally, gametocytes were immediately flushed out by removing the column from the holder, adding $5 \mathrm{~mL}$ of medium, and firmly applying the plunger supplied with the column. On average, 2.5 to 10 million gametocytes were obtained from a $25 \mathrm{~cm}^{2}$ culture flask.

\section{$\mathrm{IC}_{50}$ assays}

Compounds used for the validation included dihydroartemisinin (Sigma-Aldrich), artesunate (Apin Chemicals Limited), epoxomicin (Sigma-Aldrich), primaquine (Sigma-Aldrich), methylene blue (Sigma-Aldrich) and chloroquine (Sigma-Aldrich). The first four compounds were dissolved in DMSO, while chloroquine and methylene blue were dissolved in water.

ATP bioluminescence assay. Once purified, gametocytes were counted using a Neubauer chamber. Gametocytes $\left(5 \times 10^{4}\right.$ gametocytes per well) were transferred to a 96 -well plate in a final volume of $100 \mu \mathrm{L}$, and dilutions of each drug were added (final DMSO concentration $0.5 \%)$. Plates were incubated $\left(\right.$ at $37^{\circ} \mathrm{C}$ in a $5 \% \mathrm{CO}_{2}$ humidified incubator) for $48 \mathrm{~h}$. The ATP level of each well was determined using the BacTiter-Glo ${ }^{\text {TM }}$ reagent (Promega) according to manufacturer's recommendations. The BacTiterGlo ${ }^{\text {TM }}$ assay generates a "glow-type" luminescent signal produced by the luciferase reaction, which consists of mono-oxygenation of luciferin catalyzed by luciferase in the presence of $\mathrm{Mg} 2+$, ATP, and molecular oxygen.

Microscopic measurement of IC50 values. $\mathrm{IC}_{50}$ values were determined after $48 \mathrm{~h}$ of incubation with different dilutions of the drugs. $100 \mu \mathrm{l}$ of the culture (day 15, HT 6\%) were plated in each well with the appropriate dilution of drug. $48 \mathrm{~h}$ after, $10 \mu \mathrm{l}$ of each well was used to prepare a thin blood film and stain it with Giemsa. The number of gametocytes per 10,000-15,000 RBCs were counted and compared to the control (no drug, 0.5\% DMSO).

In vitro antiplasmodial activity on asexual stages. $\mathrm{IC}_{50}$ values were measured as previously reported [49] but all of the tests were done with serum-free cultures (using AlbuMAX II)

\section{Cytotoxicity}

Gell line. HepG2, a human caucasian hepatocellular carcinoma, was supplied by ECACG (ref. 85011430).

Routine culture. Cells were grown and maintained in EMEM (Sigma-Aldrich) supplemented with $2 \mathrm{mM}$ L-glutamine (Sigma-Aldrich) and 10\% fetal calf serum (Perbio). Cultures were maintained at $37^{\circ} \mathrm{C}$ in a humidified incubator containing $5 \%$ $\mathrm{CO}_{2}, 95 \%$ air and passages were routinely made upon reaching $80 \%$ to $90 \%$ confluence. For cytotoxicity experiments, cells were seeded onto 96 -well clear bottom black plates coated with type I collagen (Biocoat, Becton Dickinson) at a cell density of 10000 cells/well. 
Measurement of cytotoxicity. To determine cytotoxic effects, represented by the $\mathrm{IC}_{50}$ value (the concentration of drug that reduces cell viability by $50 \%$ ), cells were exposed to serial dilutions of test compounds for $48 \mathrm{~h}$ at $37^{\circ} \mathrm{C}$. The culture medium was as described above, but supplemented with $5 \%$ fetal calf serum. Following the $48 \mathrm{~h}$ exposure period, a $0.004 \%$ resazurin solution was prepared by adding $60 \mathrm{~mL}$ of Dulbecco's PBS to each resazurin tablet (VWR International). The tablet was allowed to dissolve by placing the container in a bath maintained at $37^{\circ} \mathrm{C}$, protected from light, for approximately 30 minutes. Medium was removed and $200 \mu \mathrm{L}$ of fresh culture medium and $50 \mu \mathrm{L}$ of resazurin solution were added to each well. Plates were incubated for a further $1 \frac{1}{2}$ hours. Cytotoxicity is indicated by decreased reduction of resazurin to its fluorescent product resorufin. The fluorescence was stabilized at room temperature for 15 minutes protected from light. Fluorescence was measured using a fluorescence plate reader (Victor V, Perkin Elmer) at an excitation wavelength of $515 \mathrm{~nm}$ and an emission wavelength of $590 \mathrm{~nm}$. Percentages of inhibition were calculated relative to the control wells.

\section{References}

1. Silvestrini F, Bozdech Z, Lanfrancotti A, Di Giulio E, Bultrini E, et al. (2005) Genome-wide identification of genes upregulated at the onset of gametocytogenesis in Plasmodium falciparum. Mol Biochem Parasitol 143: 100-110.

2. Tanaka TQ Williamson KC (2011) A malaria gametocytocidal assay using oxidoreduction indicator, alamarBlue. Mol Biochem Parasitol 177: 160-163.

3. Chevalley S, Coste A, Lopez A, Pipy B, Valentin A (2010) Flow cytometry for the evaluation of anti-plasmodial activity of drugs on Plasmodium falciparum gametocytes. Malar J 9: 49.

4. Adjalley SH, Johnston GL, Li T, Eastman RT, Ekland EH, et al. (2011) Quantitative assessment of Plasmodium falciparum sexual development reveals potent transmission-blocking activity by methylene blue. Proc Natl Acad Sci U S A 108: E1214-1223.

5. Peatey CL, Skinner-Adams TS, Dixon MW, McCarthy JS, Gardiner DL, et al. (2009) Effect of antimalarial drugs on Plasmodium falciparum gametocytes. J Infect Dis 200: 1518-1521.

6. Buchholz K, Burke TA, Williamson KC, Wiegand RC, Wirth DF, et al. (2011) A high-throughput screen targeting malaria transmission stages opens new avenues for drug development. J Infect Dis 203: 1445-1453.

7. Vanderberg JP, Weiss MM, Mack SR (1977) In vitro cultivation of the sporogonic stages of Plasmodium: a review. Bull World Health Organ 55: 377-392.

8. Ifediba T, Vanderberg JP (1981) Complete in vitro maturation of Plasmodium falciparum gametocytes. Nature 294: 364-366.

9. Gupta SK, Schulman S, Vanderberg JP (1985) Stage-dependent toxicity of Nacetyl-glucosamine to Plasmodium falciparum. J Protozool 32: 91-95.

10. Mann VH, Law MH, Watters D, Saul A (1996) The effects of bistratene A on the development of Plasmodium falciparum in culture. Int J Parasitol 26: $117-121$.

11. Fivelman QL, McRobert L, Sharp S, Taylor GJ, Saeed M, et al. (2007) Improved synchronous production of Plasmodium falciparum gametocytes in vitro. Mol Biochem Parasitol 154: 119-123.

12. Squatrito RC, Connor JP, Buller RE (1995) Comparison of a novel redox dye cell growth assay to the ATP bioluminescence assay. Gynecol Oncol 58: 101-105.

13. Crouch SP, Kozlowski R, Slater KJ, Fletcher J (1993) The use of ATP bioluminescence as a measure of cell proliferation and cytotoxicity. J Immunol Methods 160: 81-88.

14. Peatey CL, Spicer TP, Hodder PS, Trenholme KR, Gardiner DL (2011) A highthroughput assay for the identification of drugs against late-stage Plasmodium falciparum gametocytes. Mol Biochem Parasitol.

15. Talman AM, Blagborough AM, Sinden RE (2010) A Plasmodium falciparum strain expressing GFP throughout the parasite's life-cycle. PLoS One 5: e9156.

16. Moore LR, Fujioka H, Williams PS, Chalmers JJ, Grimberg B, et al. (2006) Hemoglobin degradation in malaria-infected erythrocytes determined from live cell magnetophoresis. Faseb J 20: 747-749.

17. Zhang JH, Chung TD, Oldenburg KR (1999) A Simple Statistical Parameter for Use in Evaluation and Validation of High Throughput Screening Assays. J Biomol Screen 4: 67-73.

18. Czesny B, Goshu S, Cook JL, Williamson KC (2009) The proteasome inhibitor epoxomicin has potent Plasmodium falciparum gametocytocidal activity. Antimicrob Agents Chemother 53: 4080-4085.

19. Benoit-Vical F, Lelievre J, Berry A, Deymier C, Dechy-Cabaret O, et al. (2007) Trioxaquines are new antimalarial agents active on all erythrocytic forms, including gametocytes. Antimicrob Agents Chemother 51: 1463-1472.
Selectivity ratio. We defined the selectivity ratio as the $\mathrm{IC}_{50}$ value in HepG2 cells $/ \mathrm{IC}_{50}$ value in P.falciparum. For a late lead or a preclinical candidate, a differential sensitivity between host and parasite at cellular level higher than 100 -fold is considered acceptable.

\section{Acknowledgments}

We wish to thank R. Sinden (Imperial College of London) and J.F. Biard for providing us with the P.falciparum 3D7HT-GFP strain and bistratene A. We are grateful to N. Bahamontes-Rosa and Pedro A. Torres for expert assistance with gametocyte culture and $\mathrm{M}$. Roncales for expert advice on gametocyte culture. We also thank David Wilson for critically reviewing the manuscript.

\section{Author Contributions}

Conceived and designed the experiments: JL MJA SL EH. Performed the experiments: JL MJA SL GM VF. Analyzed the data: JL MJA SL EH. Contributed reagents/materials/analysis tools: JL MJA SL CM VF. Wrote the paper: JL MJA EH DL.

20. Chotivanich K, Sattabongkot J, Udomsangpetch R, Looareesuwan S, Day NP, et al. (2006) Transmission-blocking activities of quinine, primaquine, and artesunate. Antimicrob Agents Chemother 50: 1927-1930.

21. Adjuik M, Babiker A, Garner P, Olliaro P, Taylor W, et al. (2004) Artesunate combinations for treatment of malaria: meta-analysis. Lancet 363: 9-17.

22. Price RN, Nosten F, Luxemburger G, ter Kuile FO, Paiphun L, et al. (1996) Effects of artemisinin derivatives on malaria transmissibility. Lancet 347 : 1654-1658.

23. Lanners HN (1991) Effect of the 8-aminoquinoline primaquine on culturederived gametocytes of the malaria parasite Plasmodium falciparum. Parasitol Res 77: 478-481.

24. Pukrittayakamee S, Chotivanich K, Chantra A, Clemens R, Looareesuwan S, et al. (2004) Activities of artesunate and primaquine against asexual- and sexualstage parasites in falciparum malaria. Antimicrob Agents Chemother 48: 1329-1334.

25. Rieckmann KH, McNamara JV, Frischer H, Stockert TA, Carson PE, et al. (1968) Gametocytocidal and sporontocidal effects of primaquine and of sulfadiazine with pyrimethamine in a chloroquine-resistant strain of Plasmodium falciparum. Bull World Health Organ 38: 625-632.

26. Smalley ME, Sinden RE (1977) Plasmodium falciparum gametocytes: their longevity and infectivity. Parasitology 74: 1-8.

27. Akoachere M, Buchholz K, Fischer E, Burhenne J, Haefeli WE, et al. (2005) In vitro assessment of methylene blue on chloroquine-sensitive and -resistant Plasmodium falciparum strains reveals synergistic action with artemisinins. Antimicrob Agents Chemother 49: 4592-4597.

28. Coulibaly B, Zoungrana A, Mockenhaupt FP, Schirmer RH, Klose C, et al. (2009) Strong gametocytocidal effect of methylene blue-based combination therapy against falciparum malaria: a randomised controlled trial. PLoS One 4: e5318.

29. Hawley SR, Bray PG, Mungthin M, Atkinson JD, O’Neill PM, et al. (1998) Relationship between antimalarial drug activity, accumulation, and inhibition of heme polymerization in Plasmodium falciparum in vitro. Antimicrob Agents Chemother 42: 682-686.

30. Mungthin M, Bray PG, Ridley RG, Ward SA (1998) Central role of hemoglobin degradation in mechanisms of action of 4-aminoquinolines, quinoline methanols, and phenanthrene methanols. Antimicrob Agents Chemother 42: 2973-2977.

31. Hoppe HC, van Schalkwyk DA, Wiehart UI, Meredith SA, Egan J, et al. (2004) Antimalarial quinolines and artemisinin inhibit endocytosis in Plasmodium falciparum. Antimicrob Agents Chemother 48: 2370-2378.

32. Basco LK, Gillotin C, Gimenez F, Farinotti R, Le Bras J (1992) In vitro activity of the enantiomers of mefloquine, halofantrine and enpiroline against Plasmodium falciparum. Br J Clin Pharmacol 33: 517-520.

33. Ringwald P, Bickii J, Basco LK (1996) In vitro activity of antimalarials against clinical isolates of Plasmodium falciparum in Yaounde, Cameroon. Am J Trop Med Hyg 55: 254-258.

34. Souri E, Nateghpour M, Farsam H, Kaji Z, Hamedi Y, et al. (2002) In Vitro Activity of Mefloquine and its Enantiomers Against Plasmodium falciparum. Iran J Pharmacol \& Therapeutics. pp 17-19.

35. Basco LK, Le Bras J (1992) In vitro activity of halofantrine and its relationship to other standard antimalarial drugs against African isolates and clones of Plasmodium falciparum. Am J Trop Med Hyg 47: 521-527. 
36. O’Neill PM, Mukhtar A, Stocks PA, Randle LE, Hindley S, et al. (2003) Isoquine and related amodiaquine analogues: a new generation of improved 4aminoquinoline antimalarials. J Med Chem 46: 4933-4945.

37. Delves M, Plouffe D, Scheurer C, Meister S, Wittlin S, et al. (2012) The Activities of Current Antimalarial Drugs on Life Cycle Stages of Plasmodium: A Comparative Study with Human and Rodent Parasites. PLoS Medicine [accepted for publication].

38. Kurth F, Pongratz P, Belard S, Mordmuller B, Kremsner PG, et al. (2009) In vitro activity of pyronaridine against Plasmodium falciparum and comparative evaluation of anti-malarial drug susceptibility assays. Malar J 8: 79.

39. Chavalitshewinkoon-Petmitr P, Pongvilairat G, Auparakkitanon S, Wilairat P (2000) Gametocytocidal activity of pyronaridine and DNA topoisomerase II inhibitors against multidrug-resistant Plasmodium falciparum in vitro. Parasitol Int 48: 275-280.

40. Butcher GA, Sinden RE (2003) Persistence of atovaquone in human sera following treatment: inhibition of Plasmodium falciparum development in vivo and in vitro. Am J Trop Med Hyg 68: 111-114.

41. Butcher GA (1997) Antimalarial drugs and the mosquito transmission of Plasmodium. Int J Parasitol 27: 975-987.

42. Ekong RM, Kirby GC, Patel G, Phillipson JD, Warhurst DC (1990) Comparison of the in vitro activities of quassinoids with activity against
Plasmodium falciparum, anisomycin and some other inhibitors of eukaryotic protein synthesis. Biochem Pharmacol 40: 297-301.

43. Sinden RE, Smalley ME (1979) Gametocytogenesis of Plasmodium falciparum in vitro: the cell-cycle. Parasitology 79: 277-296.

44. Biswas S (2001) In-vitro antimalarial activity of azithromycin against chloroquine sensitive and chloroquine resistant Plasmodium falciparum. J Postgrad Med 47: 240-243.

45. Shimizu S, Osada Y, Kanazawa T, Tanaka Y, Arai M (2010) Suppressive effect of azithromycin on Plasmodium berghei mosquito stage development and apicoplast replication. Malar J 9: 73.

46. Bregani ER, Tien TV, Monzani V, Figini G, Manenti F (2000) Azithromycin in the treatment of Plasmodium falciparum gametocytes. Preliminary observation. Panminerva Med 42: 197-199.

47. Trager W, Jensen JB (1976) Human malaria parasites in continuous culture. Science 193: 673-675.

48. Van Huyssen W, Rieckmann KH (1993) Disposable environmental chamber for assessing the drug susceptibility of malaria parasites. Trop Med Parasitol 44: 329-330.

49. Desjardins RE, Canfield CJ, Haynes JD, Chulay JD (1979) Quantitative assessment of antimalarial activity in vitro by a semiautomated microdilution technique. Antimicrob Agents Chemother 16: 710-718. 